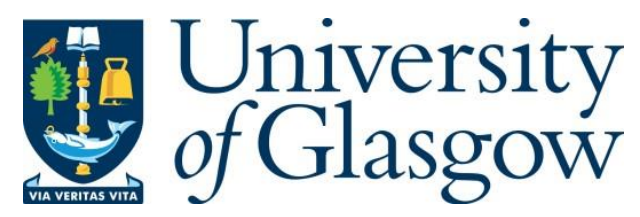

Raza, M., Awais, M., Singh, N., Imran, M. and Hussain, S. (2020) Intelligent IoT framework for indoor healthcare monitoring of Parkinson's disease patients. IEEE Journal on Selected Areas in Communications, (doi: 10.1109/JSAC.2020.3021571).

There may be differences between this version and the published version. You are advised to consult the publisher's version if you wish to cite from it.

http://eprints.gla.ac.uk/214621/

Deposited on: 22 April 2020

Enlighten - Research publications by members of the University of Glasgow http://eprints.gla.ac.uk 


\title{
Intelligent IoT Framework for Indoor Healthcare Monitoring of Parkinson's Disease Patient
}

\author{
Mohsin Raza, Muhammad Awais, Member, IEEE, Nishant Singh, Muhammad Imran, and Sajjad Hussain
}

\begin{abstract}
Parkinson's disease is associated with high treatment costs, primarily attributed to the needs of hospitalization and frequent care services. A study reveals annual per-person

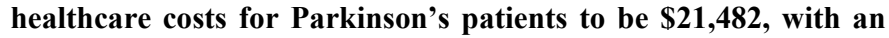
additional $\$ 29,695$ burden to society. Due to the high stakes and rapidly rising Parkinson's patients' count, it is imperative to introduce intelligent monitoring and analysis systems. In this paper, an Internet of Things (IoT) based framework is proposed to enable remote monitoring, administration, and analysis of patient's conditions in a typical indoor environment. The proposed infrastructure offers both static and dynamic routing, along with delay analysis and priority enabled communications. The scheme also introduces machine learning techniques to detect the progression of Parkinson's over six months using auditory inputs. The proposed IoT infrastructure and machine learning algorithm are thoroughly evaluated and a detailed analysis is performed. The results show that the proposed scheme offers efficient communication scheduling, facilitating a high number of users with low latency. The proposed machine learning scheme also outperforms state-of-the-art techniques in accurately predicting the Parkinson's progression.
\end{abstract}

Index Terms - Internet of things (IoT), machine learning, Parkinson's disease, probability of blocking, low latency, priority communications.

\section{INTRODUCTION AND BACKGROUND}

Neurological disorders are becoming a leading cause of disability. Among all, Parkinson's disease (PD) is one of the fastest-growing neurological disorders. PD patients worldwide have reached 6 million, with projections of 12 million by 2040[1]. PD, once a rare disorder, has become a pandemic, affecting people globally. The peculiar nature of PD also contradicts with patterns in most diseases where burden decreases with improving socioeconomic conditions. However, it increases with the improvement in socio-demographic index. Alarmingly, it has grown with the increasing per-capita Gross domestic product (GDP) in any economic evaluation [2]. In the past, our societies have been successful in confronting and arresting pandemics like HIV and breast cancer. Still, the relation of economic betterment and medical accessibility is unusual in the case of PD [3].

PD occurs due to deficiency of dopamine resulting from the loss of neurons in the substantia nigra region of the brain. There are several other cells in autonomic nervous system (ANS) which are also involved, connecting the central and peripheral regions of the brain. There are many other non-motor systems which

M. Raza is with the Department of Computer and Information Sciences, Northumbria University, UK (email: mohsinraza119@gmail.com).

M. Awais is with the Energy and Environment Institute, Faculty of Science and Engineering, University of Hull, UK (email: mawais@ieee.org).

N. Singh is with the School of Psychology, University of Birmingham, Birmingham, Birmingham, UK (email: n.singh.a@bham.ac.uk). are affected in PD and increase the overall disability in patients. The measures being taken by biologists to have a better understanding of PD are commendable. However, one of the greatest challenges faced by biologists and clinicians is the identification of biological markers that can indicate the onset and advancement of the disease in its prodromal stage [4]. Five coding mutations were identified to be causatives for PD and leading to an early onset in life. Pathway analyses of genomewide association studies (GWAS) has implicated that in pathways related to 'leucocyte or lymphocyte regulation activity', 'cytokine-mediated signaling', 'axonal guidance' and 'calcium signaling' have biological processes that can be etiological for the onset of PD in many patients [5]. Hatano et al. in 2016 [6] reported significantly lower levels of tryptophan, bilirubin and ergothioneine in PD patients and significantly higher levels of levodopa metabolites when compared to the normal controls [6]. As the elements responsible for oxidative stress in PD are not known, the metabolic activity of dopamine, mitochondrial dysfunction and neuroinflammation are all believed to play critical role of causative indicators in this disease [7]. However, the above predictive/detective measures still need to go a long way before becoming a prevalent method of PD prediction. Moreover, the clinical detections of PD and its diagnosis is an expensive affair, wherein solely dependent on marker elements which is often time consuming. Hence, in an era of advanced technologies, an alternative measure of PD prediction with use of a system of behavioral monitoring devices is the need of the hour.

With the advent and technological advancement of systemic approaches in understanding the functionality of the human body, computer scientists and engineers have started making data-driven approaches as a step towards detecting and characterizing the underlying electrophysiological changes that a PD patient's body emulates [8-10]. Clinically, accurate diagnosis of PD in the early stages is extremely complex, and the accuracy of diagnosis has been a big challenge for experts and researchers in medicine. However, the recent developments in Internet-of-things (IoT) and machine intelligence [11, 12] offer substantial benefits in detection $[13,14]$ and monitoring of patients in their early stages of PD [15-17].

Recently, attempts have been made to apply data mining and artificial intelligence-based methods on the auditory data [10, $18,19]$, human movement analysis through inertial sensors [8, 20], and imagery data [21] of the PD patients. A number of such studies are based on speech/auditory data, collected from

M. Imran is with the College of Applied Computer Science, King Saud University, Riyadh, Saudi Arabia; (email: dr.m.imran@ieee.org).

S. Hussain is with the School of Engineering, University of Glasgow, Glasgow, UK (email : Sajjad.Hussain@glasgow.ac.uk 
the PD patients. These studies established one of the important characteristics of PD, where vocal disorders were observed at an early stage, affecting the patient's speech and leading to difficulty in pronunciation of words $[22,23]$. In the past, several studies have developed machine learning-based PD detection system [24]. However, to the best of our knowledge, none of the existing systems proposed a complete physiological signal based indoor healthcare IoT paradigm for PD progression detection. It is mostly because existing systems are focused only on the data analytics of the physiological signals of individual patients instead of connecting larger cohorts of patients through IoT for remote monitoring to get better insight into PD progression. Moreover, interlinking auditory speech with motor function degradation through machine learning is still an unexplored domain. Therefore, monitoring and care taking of increasing number of PD patients is essential, where IoT can potentially lower the burden on national healthcare infrastructure. The existing machine learning based PD systems also suffer from the lack of reproducibility and generalizability [25]. This can be attributed to lack of reporting imperative information about the parameters of proposed machine learning model in the studies and lack of publicly available datasets. Therefore, the proposed work addresses these issues by analyzing the PD dataset, which is publicly available, and reporting the parameters of the developed machine learning model in the system model. In information and communications technology (ICT) infrastructure, very limited work can be found in existing IoT based large scale networks, dealing with diverse delays, reliability requirements, and communication needs. Therefore, the proposed customized IoT framework offers an efficient, cost-effective, and affordable approach for managing the chronic neurological disorders such as PD with potential to expand its applicability in diverse networks. The potential benefits of the proposed system include ease-of-use, flexibility and scalability in monitoring PD patients.

The main contributions of the proposed work are as follows:

i. A detailed system model is proposed to monitor as well as predict the progression of PD.

ii. An indoor IoT framework is proposed, which assists in taking the auditory samples of PD patients along with other fundamental sensory information, including patient's vitals and environmental parameters, which can assist in better understanding and provision of healthcare for PD patients.

iii. The work also proposes machine learning techniques to predict the progression of PD using auditory input.

The rest of the paper is organized as follows: Section II presents the proposed system model of IoT framework and machine learning techniques for PD progression detection. Section III provides results and discussion. Whereas concluding remarks and future directives are presented in Section IV.

\section{SySTEM MODEL}

PD severely affects patient's motor abilities and thus eventually requires continuous monitoring and care. Presently, most healthcare departments are suffering extensive setbacks due to a lack of qualified experts, support staff, and the availability of resources to care for the patients. The load on healthcare institutes is rising every year, and it is expected that the situation will further degrade in the next few years. PD patients require continuous monitoring and support, for which healthcare departments are not fully equipped. Therefore, the use of technology to support healthcare services by embedding smart systems for monitoring and aftercare services emerge as a viable solution to the prevailing problem.

This work proposes an IoT enabled intelligent monitoring of PD patients. The proposed work incorporates machine learning techniques to effectively support patients suffering from PD. In addition, the proposed work also aims to improve the knowledge base by introducing techniques to extensively record the phases of PD progression. The proposed work provides a complete IoT framework to support communications from different sensory elements (patients and environment) to effectively monitor PD patients. The proposed scheme can also use translational learning, whichcan be easily molded to support elderly healthcare, patient monitoring, and aftercare. Further discussion in this section is divided into 1) IoT framework and 2) Machine learning for PD progression analysis.

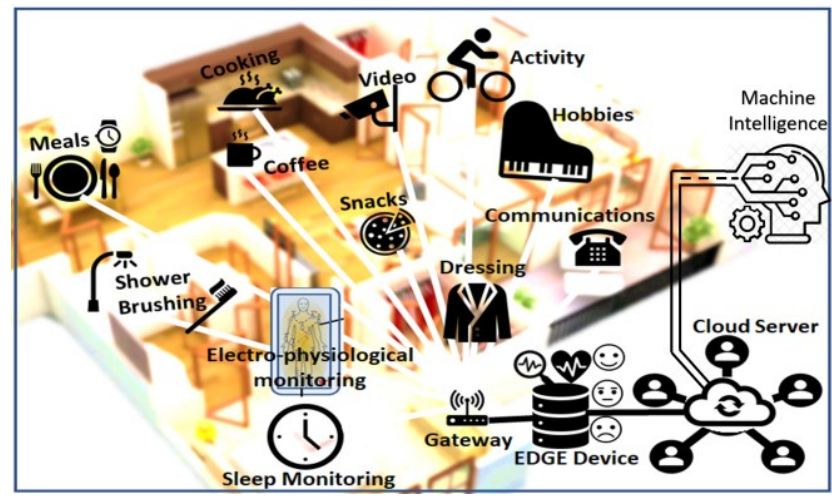

Fig. 1. IoT enabled Intelligent indoor healthcare monitoring

IoT framework supports communication among the sensors with diverse bandwidth, frequency, throughput, scheduling, and communication requirements along with edge/cloud setup for data processing and accumulation [26]. Machine learning for Parkinson's patients uses auditory information to evaluate the current progression state of $\mathrm{PD}$, which can be used as a marker to record as well as evaluate progression in patients' motor functions, and functional abilities, leading to inability score of the patient to perform desired actions. This framework is expected to feed into the knowledge base of Parkinson's progression and, thus, lead to more accurate ground truths of the progression stats in Parkinson's in future studies.

The proposed IoT infrastructure, which enables data accumulation along with edge services to pre-process and analyze the collected data, is presented in Fig. 1. For readers' convenience, frequently used system parameters are presented in Table I. The IoT framework is expected to communicate auditory input to machine learning instance on edge device [27], which analyses the progression of PD and its effects on other motor abilities of a patient. Once the progression state of PD is confirmed, the time-stamped information from other environmental and electrophysiological sensors are linked with the progression measure and stored in the database. As a proof of concept, the proposed IoT framework is thoroughly evaluated for its ability to communicate information to the IoT gateway, and a machine learning technique is evaluated, which analyses the accuracy of predicted PD state with ground truth. 
TABLE I. SyStem PARAMETERS, SYMBols AND VALUES

\begin{tabular}{|l|c|c|}
\hline Parameters & Variables & Value(s) \\
\hline IoT enabled devices & $s_{i}$ & \\
\hline Total network load & $Q$ & \\
\hline Periodic sensor network load & $\tau$ & $0.7(\%)$ \\
\hline Periodic & $P_{\Delta}$ & $\tau \times Q$ \\
\hline Non-periodic/event based & $E_{\Delta}$ & $(1-\tau) \times Q$ \\
\hline communication Time interval & $f_{i}, t_{\text {int }}$ & $25 \mathrm{~ms}$ to $1 \mathrm{sec}$ \\
\hline IoT Gateway & $G_{I}$ & - \\
\hline Superframe Duration & $T$ & $1 \mathrm{~second}$ \\
\hline Subframe duration & $T_{s}$ & $25 \mathrm{~ms}$ \\
\hline Orthogonal carrier frequency of $s_{i}$ & $f_{Q}(i)$ & - \\
\hline Total transmission slots in subframe & $n$ & 50 \\
\hline Max. non-periodic channel requests & $w$ & - \\
\hline Total Timeslots in superframe & $m$ & 2000 \\
\hline Time deadlines of cluster nodes & $\theta_{i}$ & $25 \mathrm{~ms}-1 \mathrm{sec}$ \\
\hline Timeslots in PC & $p$ & - \\
\hline Timeslots in CR & $h-p$ & - \\
\hline Timeslot duration & $t$ & $\sim 300 \mu \mathrm{s}$ \\
\hline Communications duration in timeslot & $(1-\delta) \times t$ & $\sim 250 \mu \mathrm{s}$ \\
\hline acknowledgement duration in timeslot & $(\delta) \times t$ & $\sim 50 \mu \mathrm{s}$ \\
\hline Total periodic sensor nodes & $s$ & - \\
\hline Total non-periodic sensor nodes & $b$ & - \\
\hline Total unscheduled slots in subframe & $\varphi$ & - \\
\hline Avg. delay from request to feedback & $d$ & - \\
\hline Payload & $L_{P}$ & $15.4 \mathrm{~ms}$ \\
\hline Average channel requests & $\lambda$ & - \\
\hline Blockage probability & $P_{b}$ & - \\
\hline Scheduled slots in subframe & $\xi_{s}$ & - \\
\hline Free slots in subframe & $\xi_{f}$ & - \\
\hline Priority levels High, Medium, Low & $P_{\text {high }}, P_{m e d}, P_{l o w}$ & - \\
\hline
\end{tabular}

\section{A. Proposed IoT-based Patient Monitoring system}

In the proposed work IoT enabled devices $\left(s_{i}\right)$ are assumed where the initial framework of IEEE802.15.4e is used which allows communications after regular intervals. However, in the given scenario, the information is communicated in both periodic $\left(P_{\Delta}\right.$, i.e. after regular intervals) and event-driven $\left(E_{\Delta}\right)$ fashion. In addition, communication periodicity $\left(f_{i}\right)$ for sensor $\left(s_{i}\right)$ might be different from communication periodicity $\left(f_{j}\right)$ for sensor $\left(s_{j}\right)$ for $(i \neq j)$, where communications from $s_{i}$ and $s_{j}$ take place after every $t_{i}$ and $t_{j}$ intervals respectively (given $t_{i}=$ $\left.1 / f_{i} \& t_{j}=1 / f_{j}\right)$. Therefore, a scheduling algorithm is proposed to not only manage periodically communicating sensor nodes but also communications from dynamic and event-driven sensor nodes. The scheduling algorithm allows communications from all sensors communicating periodically in an orderly fashion where a static schedule is developed for all periodic communications along with instantaneous dynamic scheduling of on-demand communications. In addition to this, a priority enabled communication infrastructure for requests from on-demand/event-driven communication sources is also proposed. A suitable priority of access is maintained in the proposed IoT system to allow effective communications from event-driven devices to give timely channel access, analysis, and feedback.

\section{1) Clustering, gateway and IoT infrastructure}

It is assumed that the IoT devices/sensors are affiliated to IoT Gateway $\left(G_{I}\right)$ in star topology during the setup phase. Time Division Multiple Access (TDMA) and beacon-enabled communications are used, which limit the interference of different devices accessing the channel at the same time. Thus, mitigating the packet collision commonly seen in Carrier Sense Multiple Access (CSMA) and other collision avoidance schemes. To enable timely communications from audio, video and sensory nodes in the IoT infrastructure, information is transmitted in short subframes, each of duration $T_{s}$ [28]. As represented in Fig. 2, a sub-frame is further divided in four sections: beacon (B), channel request (CR), scheduled periodic communications (PC), and the scheduled requested communications (SRC). Beacon synchronizes all the local clocks of sensor nodes with $G_{I}$. CR allows on-demand communication requests to be made by non-periodic sensor nodes and scheduled by $G_{I}$. Any channel requests by nonperiodic sensor nodes are made directly to the gateway [29]. The total number of non-periodic nodes is expressed by $w$. Note that the sensor nodes in the indoor environment are allocated a unique orthogonal carrier $\left(f_{Q}(i), i=1 \rightarrow w\right)$, which is broadcasted by the sensing devices during CR period. These orthogonal frequency bands are distributed in $\epsilon$ priority levels, where more critical sensor node is assigned higher priority carrier. Upon reception of carriers during $\mathrm{CR}, G_{I}$ distinguishes between the transmitted frequency bands, thus identifying and

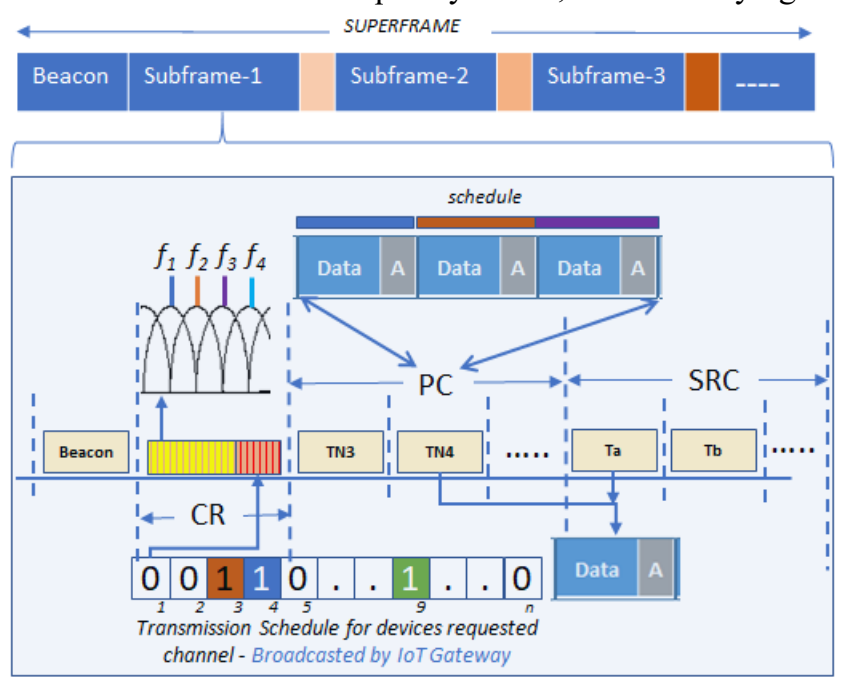

Fig. 2. Superframe structure

scheduling more critical requests first. The schedule of transmissions is broadcasted by the gateway to confirm acceptance or rejection of channel requests, as represented in Fig. 2, transmission schedule. During PC and SRC, periodic and non-periodic communications take place.

Each subframe allows communication of a maximum of $n$ sensor nodes. The communications of each sensor node except audio-visual inputs is completed in one timeslot of duration $t$. A timeslot is subdivided in 'communication' and 'ack' window of duration $(1-\delta) \times t$ and $\delta \times t$, respectively. The communications frequency/periodicity of periodic sensor node $i\left(s_{i}\right)$ is given by $\theta_{i}$, and average channel requests per unit time for event-driven/non-periodic sensors is given by $\lambda$.

All channel access requests from non-periodic sensors are made prior to communications of every subframe, which allows the system to broadcast the communication schedule before subframe communications are initiated. The proposed scheduling scheme offers a static schedule for periodic communications, allocating timeslots as evenly as possible in each subframe. Whereas, the free timeslots in each subframe 
are filled with dynamic channel requests received from nonperiodic sensors. This scheduling of free timeslots is carried out by a dynamic scheduling algorithm, which also considers the priority of the requesting sensor nodes.

Communications between the sensor nodes and IoT gateway is carried out in subframes where multiple subframes formulate a superframe. The duration of the subframe and superframe is derived from the communication interval of periodically communicating nodes. For instance, if the shortest interval for one of the sensor nodes is 20 milliseconds (ms) (i.e., it has to communicate its data to the gateway, $G_{I}$, every $20 \mathrm{~ms}$ ), the subframe duration will be selected $20 \mathrm{~ms}$. Whereas, in the same cluster, if a sensor node has to communicate to $G_{I}$ every 400 $\mathrm{ms}$, which is the longest interval, then the superframe duration would be $400 \mathrm{~ms}$. In general, subframe and superframe duration are expressed as $T_{S}$ and $T$ respectively where $T=\frac{1}{\theta_{i}(\min )}$ and $T_{S}=\frac{1}{\theta_{i}(\max )}$. However, slight variations in the duration of $T_{S}$ and $T$ are introduced such that $\forall u \in \mathbf{I} \mid T=u \times T_{s}$ is satisfied. The information accumulated from sensor nodes at $G_{I}$ during the superframe is relayed to the edge device $\left(e_{I}\right)$, which at the first instance assigns a unique id to the patients for anonymity purposes. The accumulated data is processed at edge device where data is extracted, preprocessed, labeled, and anonymized before its further propagation to central cloud space. It is assumed that the data analytics and visualization of the accumulated cloud data are used to assist in monitoring, prescription, and diagnosis of the current state of PD patients.

2) Scheduling Algorithm

As stated earlier, the scheduling algorithm schedules both periodic and non-periodic communications. The scheduling is achieved in two phases. At first, static scheduler schedules periodic communications within the cluster. The periodic communications are although communicated after regular intervals, yet, the interval duration for each sensor can vary significantly. In Fig. 3, the time interval of communications of each sensor is presented. The bar graphs in Fig. 3 present the periodicity (regular intervals after which the communications have to be made by each node). For instance, sensor node 1 needs to resend/communicate its readings every 1 second, whereas node 30 has to communicate every 0.2 seconds. The represented communication deadlines of each sensor are quite randomly distributed between $0-1$ seconds. To schedule these periodic communications, the static scheduler defines superframe and subframe duration. Due to the high complexity of scheduling heterogenous deadlines, all the divisors of time duration $T$ are listed and heterogeneous time interval/time deadlines of each node are approximated to the nearest lower divisor, $\theta_{i}$ (see algorithm 1 Line:1). The duration of superframe and subframe is defined using $\theta_{i}(\mathrm{~min})$ and $\theta_{i}(\max )$ (see algorithm 1 Line: 2, 3).

The scheduling algorithm considers five categories: $C_{1}$ to $C_{5}$ (scheduled in algorithm 1 Line:8-14). It is noteworthy that the static schedule produced in Algorithm 1 only schedules $C_{1}$ to $C_{4}$ whereas $C_{5}$ is scheduled in real-time. Scheduling of $C_{1}$ considers nodes with interval/deadline $\left(t_{\text {int }}\right)$ equal to $T_{s}$. $C_{2}$ considers nodes with $t_{\text {int }}=u \times T_{s} \mid u \in \mathbf{I} \& u$ is even \& $u \times$ $T s \leq T / 2 . \quad C_{3}$ considers nodes with $t_{\text {int }}=u \times T_{s} \mid u \in$ I \& $u$ is odd \& $u \times T s \leq T / 2 . C_{4}$ considers nodes with $t_{\text {int }}=$
T. $C_{5}$ considers non-periodic nodes and schedules them in realtime. In the proposed scheme, the load from periodic sensor nodes is assumed to be maximum of $\tau$ percent of the total load where total timeslots in superframe are given by

$$
m=\left(T / T_{s}\right) \times n
$$

The overall required timeslots by periodic sensors is given by

$$
\beta=\left\{\left(\sum_{i=1}^{s} b_{i} \times S\left(r_{i}, s_{d l}\right)\right) \times T\right\} / B_{\Delta}
$$

where $s$ is the total number of periodic sensor nodes, $b_{i}$ is the number of bits to be transmitted by source $i$ in every communication, $r_{i}$ is the communication time interval of the node $i, B_{\Delta}$ is total bits communicated in one timeslot and $s_{d l}$ is the vector consisting of all possible symmetric deadlines (divisor of $T$ ). The $S\left(r_{i}, s_{d l}\right)$ allows symmetrical attributes of the superframe by approximating $r_{i}$ to the nearest symmetrical deadline from the $s_{d l}$ vector.

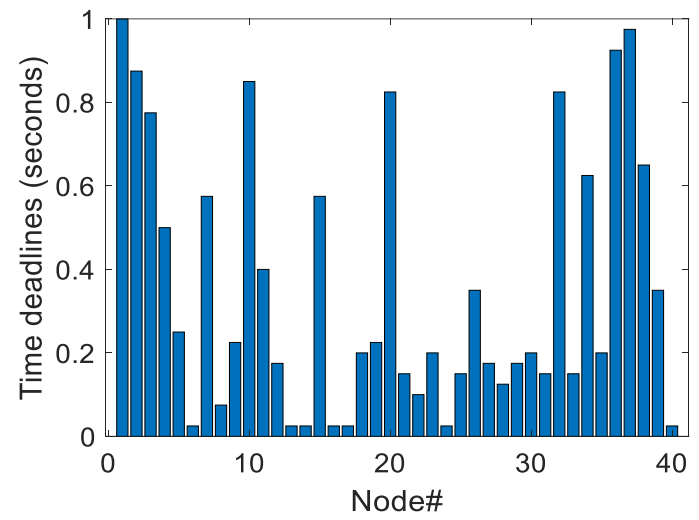

Fig. 3. Periodic communication interval of different sensor nodes

Apart from scheduling the periodic communications, the scheduling algorithm also considers non-periodic communications and schedules them in real-time. The scheduling algorithm is run on $G_{I}$.

3) Resource management and Dynamic Design

In the proposed system, while the periodic sensor nodes receive a static schedule; however, the behavior of non-periodic nodes need to be carefully modelled to keep the blocking probability of nodes below the desired thresholds. In addition, the delay must also be carefully modelled to evaluate the accurate performance of the proposed IoT infrastructure. This subsection particularly focuses on mathematical modelling of the proposed system to analyze the blocking probability $\left(P_{b}\right)$ of IoT channel and to suggest a maximum number of non-periodic IoT nodes suitable for maintaining $P_{b}$ below certain thresholds. The average on-demand communication requests $(\lambda)$ are also considered in system modelling.

The scheduling of channel requests primarily depends on the available/unscheduled timeslots in a subframe $(\varphi)$. The unscheduled slots $(\varphi)$ in each subframe express the available resources. Since the resource, $\varphi$, is limited, a finite number of sensor nodes can be accommodated while ensuring certain $P_{b}$, given a certain arrival rate $(\lambda)$ is expected. Individual sensor nodes are modelled as poison distribution where its conditional probability mass function (PMF) can be expressed as

$$
P_{I}(i)=\left\{\begin{array}{cc}
\frac{\left(\lambda T_{s}\right)^{i} e^{-\lambda T_{s}} / i !}{\sum_{j=0}^{w} \lambda T_{s}{ }^{j} e^{-\lambda T_{s}} / j !} & \text { where } i=0,1,2, \ldots, w \\
0 & \text { otherwise }
\end{array}\right.
$$


where $\lambda$ is the average number of requests, $T_{s}$ is the unit time, which in this case is taken equal to subframe duration, and $w$ is the maximum number of requesting devices.

The average delay $(d)$ is modeled as a function of average requests per unit time $(\lambda)$, average timeslots available $(s)$ per subframe, and channel conditions. The mathematical notation for the average delay, $d$ for a given payload, $L_{p}$, is as follows:

$$
\begin{array}{r}
d=\left(\left(\frac{\sum_{i=1}^{S} i \times\left(\lambda T_{s}\right)^{i} e^{\left(\lambda T_{s}\right)} / i !}{\sum_{j=0}^{S}\left(\lambda T_{s}\right)^{j} e^{\left(\lambda T_{s}\right)} / j !}+(n-s)\right) \times t\right) \\
+\left(T_{s} \times \sum_{i=1}^{s}\left(\begin{array}{l}
S \\
i
\end{array}\right) p^{i}(1-p)^{s-i}\right)+L_{p}
\end{array}
$$

The probability of blockage $\left(P_{b}\right)$ of communications for nonperiodic sensor nodes highly depend on the average number of available slots $(\varphi)$ in each subframe, the number of nonperiodic sensor nodes $(b)$, and the average number of requests per sensor node per unit time $(\lambda)$. Therefore, in the given scenario, $P_{b}$ can be expressed as

$$
P_{b}=\sum_{i=\varphi+1}^{w} \frac{\left(\lambda T_{s}\right)^{i} e^{-\lambda T_{s}} / i !}{\sum_{j=0}^{w} \lambda T_{s}^{j} e^{-\lambda T_{s} / j !}}
$$

Further discussion on scheduling, delay, and blockage probability is continued in Section III section.

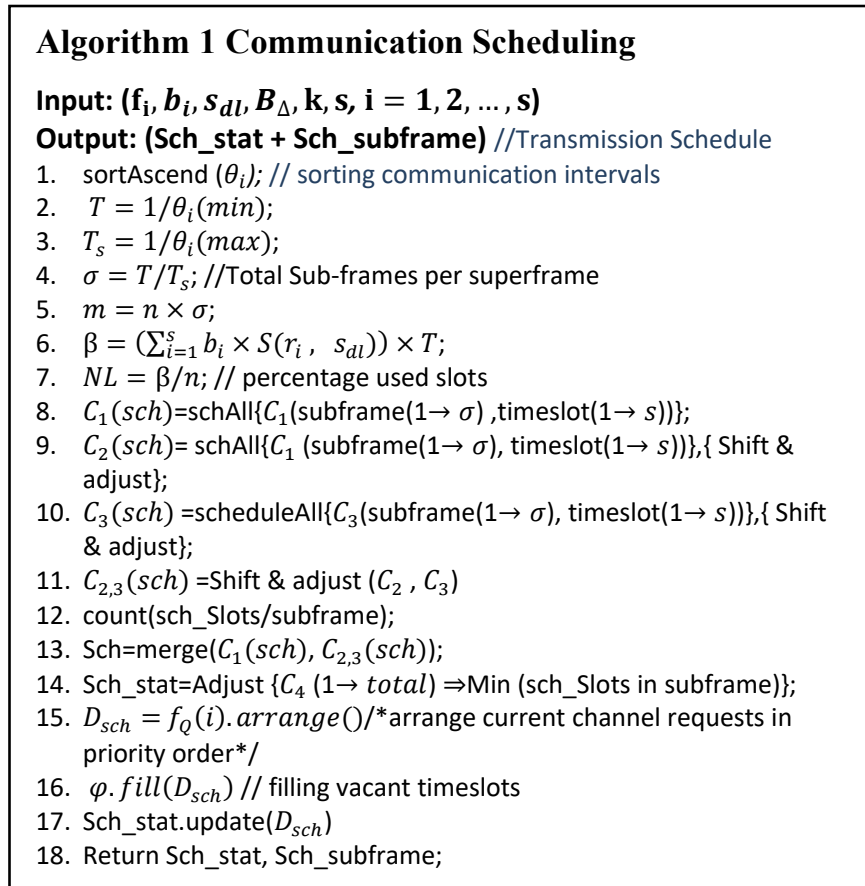

The use of IoT framework allows near real-time data communications, thus enabling large-scale data assembly from Parkinson's patients. Though, the data collection from different sensory elements in controlled environments is very important, yet, the machine learning plays a vital role in devising strategies to not only process large data but also to label the biological markers with the help of well-established speech based Parkinson's progression analysis. Further details of the proposed machine learning techniques are covered as follows.

\section{B. Machine Learning Model Development for Parkinson's Disease Progression Prediction}

\section{1) Dataset}

This work utilizes a dataset collected by Tsanas et al. [30] using At Home Testing Device (AHTD) from intel corporation [31]. The ATHD is capable of recording PD measures related to dexterity (i.e., the tasks related to fine motor skills muscles) and speech. The dataset analyzed here recorded only speech-related task where subjects were instructed to perform sustained phonation of vowels. The study then used the Unified Parkinson Disease Rating Scale (UPDRS) to quantify the severity of the disease, where speech recordings were obtained through the ATHD device and were mapped to predict the UPDRS score. The UPDRS score is a clinical measure that provides the severity of the disease. UPDRS scale ranges from 0 to 176 , where 0 represents healthy adults, and 176 represents completely disabled individuals. This scale considers the activities of daily living, mental behavior, mood, and motor activities [30]. Another variant of this scale is also measured in this study, called the motor UPDRS scale. It ranges from 0 to 108, where 0 represents physically active and healthy individuals, and 108 represents severe motor impairments and tremors. The UPDRS serves as a ground truth measure in this study due to its strong correlation with the severity of the PD in clinical settings [30].

Forty-two subjects suffering from idiopathic PD participated in data collection procedures and performed voice recordings at their residential settings using AHTD. The dataset was collected over a period of six months, where voice recordings were captured once every week on a specified day. The data collection procedure was specified to the subjects on how and when to perform the experiment. The data collected through each of the ATHD devices was sent to the clinic, which was then used to obtain the UPDRS score and to perform further processing on auditory data. The subjects performed two sets of voice recordings: 1) carrying on the sustained vowels phonation, 2) describing the static photographs through running speech. The sampling frequency of the device was $24 \mathrm{kHz}$. This resulted in a total of 5875 signals recorded by 42 subjects over a period of six months.

TABle II: FEATURE ExtRActed From Voice ReCORdings [30]

\begin{tabular}{|l|l|}
\hline Sr. & Computed feature description \\
\hline 1 & KayPentax multidimensional voice jitter in percentage \\
\hline 2 & KayPentax multidimensional voice jitter in microseconds \\
\hline 3 & KayPentax multidimensional voice amplitude perturbation \\
\hline 4 & KayPentax multidimensional voice average perturbation quotient \\
\hline 5 & Jitter difference divided by the average period \\
\hline 6 & KayPentax multidimensional voice local shimmer \\
\hline 7 & KayPentax multidimensional voice in dbs \\
\hline 8 & Shimmer 3-point perturbation quotient \\
\hline 9 & Shimmer 5-point perturbation quotient \\
\hline 10 & KayPentax multidimensional voice 11 point perturbation quotient \\
\hline 11 & Shimmer amplitude difference over period \\
\hline 12 & Noise to harmonic ratio \\
\hline 13 & Harmonic to noise ratio \\
\hline 14 & Recurrent period density entropy \\
\hline 15 & Detrended fluctuation analyses \\
\hline 16 & Pitch period entropy \\
\hline
\end{tabular}




\section{2) Feature Extraction}

The features used in this study are similar to the one extracted by Tsanas et al. [30]. The features extracted are referred to as dysphonia measures. A variety of dysphonia measures were extracted from the speech signals, and each of these dysphonia measures represented a number as a feature value that described the unique characteristics of the signal. A total of 16 dysphonia measures were used in this study as these measures had a maximum correlation with the motor UPDRS and overall UPDRS scores [30]. These measures were extracted from the voice recording obtained through the AHTD device, as listed in Table II. Each recording resulted in 16 features. Therefore, 5875 signal recordings obtained through 42 subjects resulted in a feature matrix of 5875 (samples) x16 (features).

3) Machine Learning Model development to predict

\section{Parkinson's Disease progression}

The secondary objective after the successful development of the IoT framework is to predict the PD progression through auditory recordings captured by AHTD. The UPDRS and motor UPDRS scores are used to validate the performance of the proposed voice recording based PD progression detection system over a period of six months. In the proposed work, extreme gradient boosting (XGB) is used as a regression method [32, 33] to develop a machine-learning based PD progression detection model using the feature-set extracted from the voice recording of ATHD device. The performance of this model is then validated with the ground truth (i.e., motor UPDRS score and overall UPDRS score).

The objective function of XGB is shown in Eq. 1 [34]. The loss $\left(l^{(t)}\right)$ function aims to reduce the predictor error between the true and the predicted outcomes.

$$
l^{(t)}=\Omega\left(f_{t}\right)+\sum_{i=1}^{h} l\left(y_{i-\text { true }}, y_{i-\text { pred }}^{(a-1)}(a)+f_{a}\left(X_{a}\right)\right)
$$

here $f$ describes the total structures in the regression tress with weights, $i$ represente the data samples, $t$ represents the iteration, $X$ represents the feature set, $h$ represents the total number of data samples, $y_{i \text {-pred }}$ represents the predicted UPDRS values of the proposed machine learning model and the $y_{i-t r u e}$ represents the true or the actual values of the UPDRS scale obtained in clinical settings.

$$
\Omega\left(f_{a}\right)=\gamma \mathrm{W}+0.5 \times \delta\|\mathrm{w}\|^{2}
$$

The first term in Eq. 7 describes the complexity of the model and the second $(\delta)$ is the regularization which prevents the model from overfitting, $W$ represents the number of leaves per tree and $\mathrm{w}$ represents the weight of the leaf.

The mean absolute error (MAE) is computed as a performed measure which describes the predictor error of (Eq. 6). The large value of MAE corresponds to high prediction error and a larger deviation from true values, and vice versa.

$$
M A E=1 / h \sum_{i=1}^{h}\left|y_{i-p r e d}-y_{i-\text { true }}\right|
$$

The 90/10 split based cross-validation procedure is used for the XGB based machine learning model development and performance evaluation. The classifier model is trained using $90 \%$ of the data samples ( 5288 data samples) and tested on the remaining $10 \%$ (587 data samples) for performance evaluation and validation. This process is repeated for 1000 iterations by randomly assigning the testing and training samples for cross- validation. The validation and performance evaluation adopted is the same as the one performed by Tsanas et al. [30] to provide a fair and unbiased comparison. The parameters used for XGB classifier are: objective function $=$ mean squared error, number of estimators $=100$, learning rate $=0.08$, gamma $=0.5$, maximum depth, minimum child weight $=7$.

\section{RESULTS AND DISCUSSION}

\section{A. Performance Analysis of IoT infrastructure}

The primary objective of the proposed IoT based solution for data accumulation and analysis of the progression of Parkinson's is to minimize the need for frequent external interventions and to sustain healthy living in the patients. The proposed scheme receives the data from a variety of sensors and records auditory feedback from the patients to analyze the progression of the disease. The proposed IoT infrastructure is aimed at managing communications from diverse sources with desynchronized periodicity, which is achieved using the scheduling algorithm. The scheduling algorithm is evaluated based on its ability to schedule a large number of nodes along with minimum possible variation in the scheduled slots $\left(\xi_{s}\right)$ to free slots $\left(\xi_{f}\right)$ ratio in each subframe. For evaluation purposes, a subframe duration of $25 \mathrm{~ms}$ and a superframe of the duration of 1 second is used. Number of nodes with periodic communication requirements which are affiliated to $G_{I}$, are varied from 20 to 80 . Bandwidth requirements of the sensor nodes is also changed depending on the type of the sensor (i.e., whether it is a binary sensor, audio, or video feedback). As represented in Fig. 4, the scheduling algorithm schedules complex communications with high efficiency. The right-hand side $\mathrm{Y}$-axis represents the percentage timeslots scheduled in each subframe (represented by bar plot), which shows that up to 70 sensor nodes can be effectively scheduled by the scheduling algorithm, keeping approximately $25 \%$ free timeslots in each subframe for non-periodic communications. In the Figure, the left-hand $\mathrm{Y}$-axis shows the average number of timeslots $(n=50)$ scheduled per subframe along with the deviation (represented as error bar plots). The relatively small deviation from the mean depicts high accuracy of the scheduling algorithm.

The scheduling of non-periodic nodes is dependent on the available resources (i.e., $\xi_{f}$ per subframe). It also depends on the expected average number of channel requests per sensor node per second. The analysis presented here covers a relatively high number of requests per second (upto $\lambda=20$ ). As represented in Fig. 5, for a relatively low share of available resources $\left(\xi_{f}=5\right.$, i.e., $10 \%$ of the given resources), a relatively bearable probability of blockage $\left(\mathrm{P}_{b}<3 \%\right)$ is achieved for up to 10 non-periodic sensor nodes with, on average, ten channel requests per second. Thus, the proposed system can accommodate 85 sensor nodes with 75 periodic sensor nodes with $\mathrm{P}_{b}=0$, and ten non-periodic sensor nodes with $\mathrm{P}_{b}<3 \%$. Please note that the periodic and non-periodic nodes are assigned $90 \%$ and $10 \%$ resources. In Fig. 6, a 70/30 resource share is allocated to periodic and non-periodic sensor nodes. It can be seen from Fig. 4 and Fig. 6 that this accommodates 60 periodic sensors with 40 non-periodic sensors with $\mathrm{P}_{b}<5 \%$ for average requests per node as high as 10 per second. If the 
average channel requests for non-periodic sensors is reduced to 5 per second (i.e., on average a channel request is made by each individual sensor node every $200 \mathrm{~ms}$, a more realistic scenario) the number of non-periodic nodes that can be accommodated with proposed system reaches to 200 (in addition to 60 periodic sensors), which confirms the suitability of proposed IoT framework for not only the home patients but also for hospital wards and care homes.

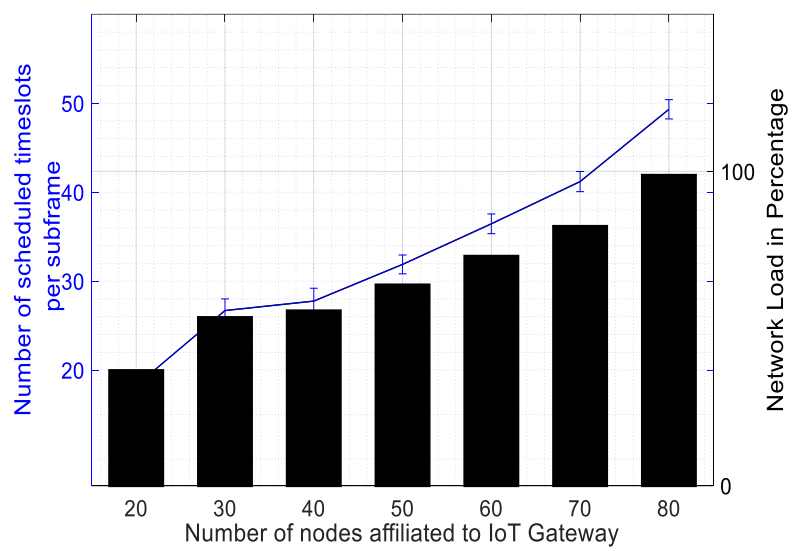

Fig. 4. Average scheduled timeslots in subframe for periodic communications.

In addition to the probability of denial of service/blockage probability, the delay is also an important attribute to evaluate the performance of the proposed work. Since most of the healthcare scenarios also have some level of emergency involved, therefore, channel assignment delay is evaluated from the time of the request to the assignment of timeslot. For evaluation purposes three levels of priority $\left(P_{\text {high }}, P_{\text {med }}, P_{\text {low }}\right)$ are considered among the requesting nodes, as presented earlier in Fig. 2 and Section 3. In evaluation, it is assumed that $30 \%$ of resources are reserved for non-periodic communications. In addition, the probability of denial of service of less than $5 \%$ $\left(\mathrm{P}_{b}<5 \%\right)$ is maintained. The average delay for three priority classes of sensor nodes is evaluated where affiliated nonperiodic nodes are varied from 10 to 200 and $\lambda=5$. As represented in Fig. 7, the delay for high priority sensor node ( $10 \%$ of total sensors) is relatively low with average channel access delay of nearly $11 \mathrm{~ms}$, which is well within the requirements of any health-related emergency system. The delay of medium priority sensor nodes is also relatively low; however, saturation is approached for low priority, $P_{\text {low }}$ when nodes exceed 120, after which delay rises notably. A similar pattern in delay arises for $P_{\text {med }}$ as well after nodes are increased over 250. However, the overall performance of the system is well suited for the proposed scenario where high priority nodes can timely access the channel for critical communications.

The proposed IoT framework and scheduling algorithm not only offers improved data scheduling but also allows timely communications of the periodic data originating from different sources. The thorough analysis of the proposed framework suggests the suitability of IoT infrastructure for information collection and remote monitoring. However, for a system to be completely autonomous and to be able to operate independently, the accuracy of the machine learning and AI- driven diagnosis in healthcare is very important. The following discussion covers the performance of AI in detail.

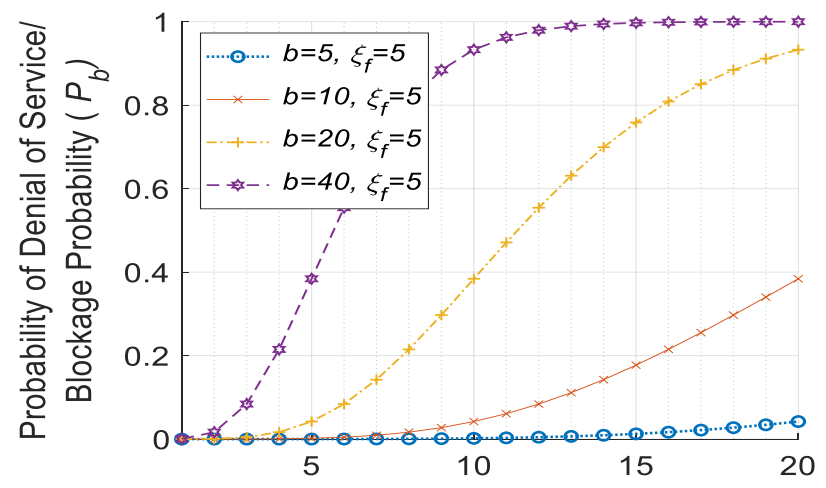

Number of requests per node per second

Fig. 5. $\mathbf{P}_{\mathbf{b}}$ with changing requests per node per second $(\lambda)$

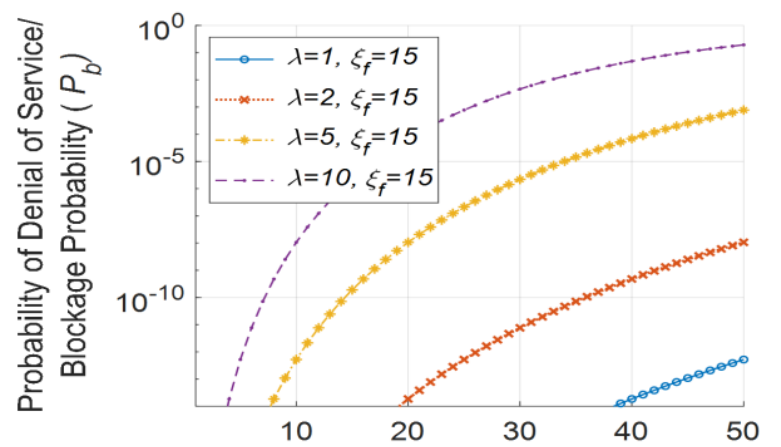

Number of non-periodic nodes affilited to $G_{I}$

Fig. 6. $\mathbf{P}_{\mathbf{b}}$ as a function of affiliated non-periodic nodes

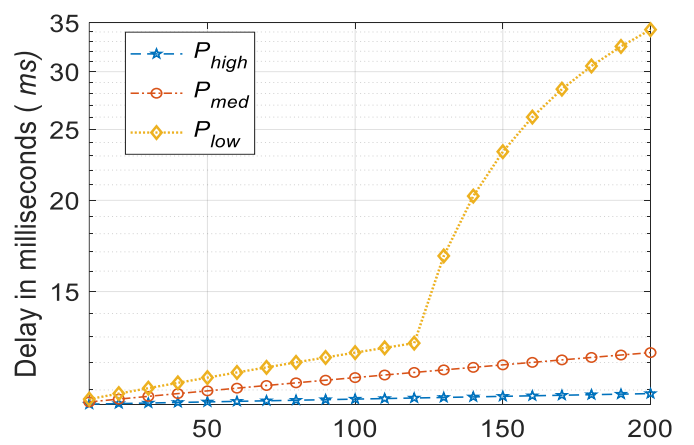

Number of non-periodic nodes requesting Channel

Fig. 7. Delay analysis of priority enabled communications in loT

\section{B. Performance Analysis of PD progression detection}

The secondary objective of the proposed model is to accurately predict PD progression through machine learning. The machine learning model was trained using the features-set obtained from auditory recording and validated using the ground truth measure obtained from the clinical setting (motor UPDRS scale and overall UPDRS scale). The findings of the proposed PD progression prediction through overall UPDRS and motor UPDRS are presented in Fig. 8(a). The findings are presented for the training and testing scenarios using MAE as a performance metric. The mean and standard deviation of MAE was calculated over 1000 iterations for both training and testing. The performance analysis shows that the proposed XGB based method is capable of predicting the motor UPDRS score with MAE of $2.29 \pm 0.04$ and $5.09 \pm 0.16$ on the training and 
testing datasets respectively. Moreover, the proposed method resulted in MAE of $2.97 \pm 0.05$ and $6.45 \pm 0.21$ in the training and testing datasets respectively to predict the overall UPDRS score.

These findings show the strength of the proposed method in predicting the PD progression accurately through dysphoniabased measures obtained from voice recordings with low MAE. A comparative analysis of MAE in predicting the PD progression is also performed among the proposed method and the method developed by Tsanas et al. [30]. The study provides a fair and unbiased comparison as both methods have used the same dataset, cross-validation procedure, and the performance metric. The MAE errors of both studies in predicting the motor UPDRS and overall UPRDS through voice recordings are depicted in Fig. 8(b).

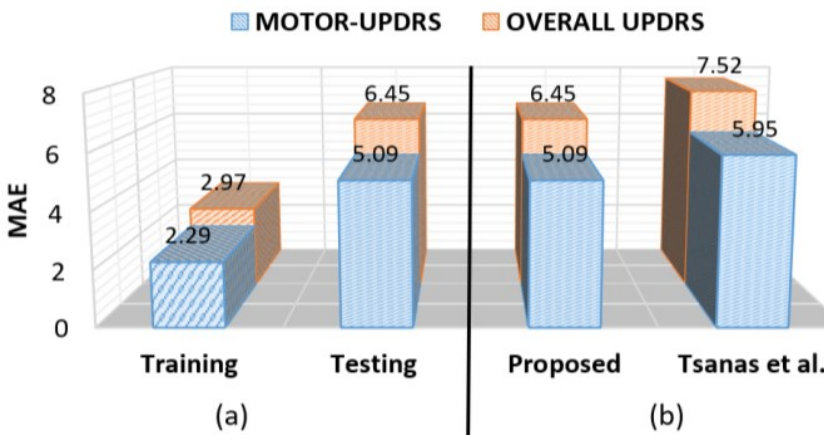

Fig. 8: MAE Analysis in Predicting PD Through UPDRS Scores (a) Proposed (b) Comparison: proposed with Tsanas et al.

The results show that the proposed system has outperformed the PD progression detection method proposed by Tsanas et al. [30], and MAE is reduced significantly both in motor UPDRS prediction as well as overall UPDRS prediction. These findings are quite interesting and show the significance of the proposed method in accurately predicting the PD progression over time.

For better visualization and understanding, the PD progression prediction of a single patient, over six-month duration through motor UPDRS scale, and overall UPDRS scale is depicted in Fig. 9.

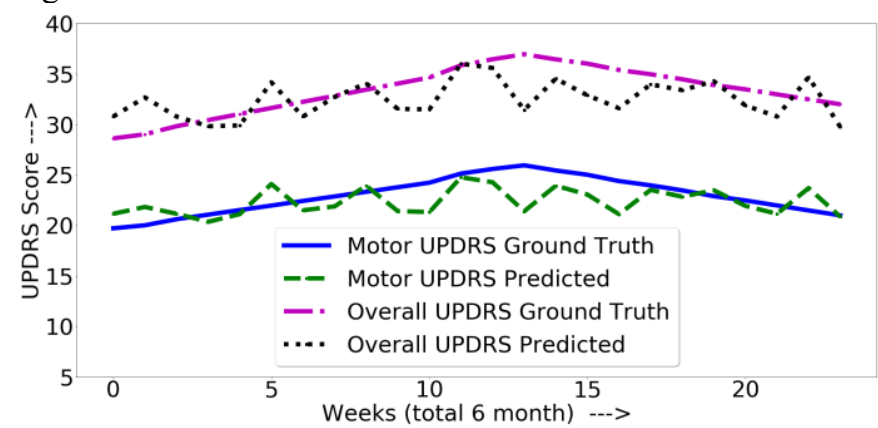

Fig. 9. UPDRS score prediction of proposed method w.r.t ground truth

The graphs present the actual values of UPDRS or the ground truth values obtained through clinical settings and the UPDRS prediction performed by our proposed XGB based method. It is quite evident from these plots in Fig. 9 that the proposed method can efficiently track both PD progression scales (motor UPDRS, overall UPDRS) with high efficiency and low MAE. These findings also suggest that sustained vowel phonation audio recording offers potential for tracking PD progression remotely with high accuracy.

\section{CONCLUSION}

The proposed IoT and machine learning framework aims to offer an effective solution to support the routine life of Parkinson's patients with minimal external interference. It is also aimed at providing a framework to enable continuous monitoring and progression analysis of PD. The proposed IoT infrastructure allows continuous monitoring of electrophysiological and environmental parameters of an indoor environment. It also incorporates a large number of sensors, which demonstrates its ability to function in hospital wards and care homes. The results show that the proposed framework not only offers efficient communication scheduling but also enables prioritized channel access. A relatively low average delay of $11 \mathrm{~ms}$ was observed for high priority sensor nodes. In addition, the scheduling algorithm can handle up to 250 sensor nodes (periodic and non-periodic), which can be scaled to even larger networks with suitable clustering schemes. The probability of denial of service was also limited to $5 \%$. The proposed machine learning algorithm also offers high accuracy of PD progression prediction.

While the proposed work offers notable improvements, however, it only utilizes voice recordings based measure to develop PD progression prediction system. In addition, it does not cover how inertial sensor-based methodologies and other human movement capturing systems can assist in predicting the PD progression. Therefore, as a future direction, it would be interesting to perform a comparative study that observes the performance of the proposed voice recording based PD progression detection and the inertial sensors-based PD progression detection. The functionalities of edge and cloud devices can be further explored. In addition to this, a long-term evaluation and logging of sensory data with auditory UPDRS predication can also improve the knowledge base and understanding of progression stages in PD.

ACKNOWLEDGEMENT

Imran's work is supported by the Deanship of Scientific Research through research group project number RG-1435051.

\section{REFERENCES}

[1] V. L. Feigin et al., "Global regional and national burden of neurological disorders during 1990-2015: a systematic analysis for the Global Burden of Disease Study 2015", Lancet Neurol, vol. 16, no. 11, pp. 877-897, 2017.

[2] E. Dorsey, T. Sherer, M. Okun and B. Bloem, "The emerging evidence of the Parkinson pandemic, "J. Parkinson's Disease, vol. 8, no. s1, p. S3S8, 2018.

[3] S. Bohingamu Mudiyanselage et al., "Cost of living with Parkinson's disease over 12 months in Australia: a prospective cohort study," Parkinson's Disease, vol. 2017, 2017.

[4] W. Poewe et al., "Parkinson disease". Nature reviews Disease primers, 2017;3:17013 Epub 2017/03/24. doi: 10.1038/nrdp.2017.13.

[5] J. Brás, R. Guerreiro, and J. Hardy, "SnapShot: genetics of Parkinson's disease," Cell, vol. 160, no. 3, pp. 570-570. e1, 2015.

[6] T. Hatano et al., "Identification of novel biomarkers for Parkinson's disease by metabolomic technologies," J. Neurol Neurosurg Psychiatry, vol. 87, no. 3, pp. 295-301, 2016.

[7] J. Blesa, I. Trigo-Damas, A. Quiroga-Varela, and V. R. Jackson-Lewis, "Oxidative stress and Parkinson's disease," Frontiers Neuroanatomy, vol. 9, p. 91, 2015. 
[8] M. Corzani, A. Ferrari, P. Ginis, A. Nieuwboer, and L. Chiari, "Analysis of Biofeedback Effects in Parkinson's Disease at Multiple Time-Scales," in Intl. Conf. on NeuroRehabilitation, pp. 815-818, 2018.

[9] R. R. Guimaraes et al., "Intelligent network security monitoring based on optimum-path forest clustering," IEEE Network, vol. 33, no. 2, pp. 126$131,2018$.

[10] D. Gupta et al., "Optimized cuttlefish algorithm for diagnosis of Parkinson's disease", Cogn. Syst. Res., vol. 52, pp. 36-48, Dec. 2018.

[11] M. M. Mahmoud et al., "Enabling technologies on cloud of things for smart healthcare," IEEE Access, vol. 6, pp. 31950-31967, 2018.

[12] J. J. Rodrigues et al., "Enabling technologies for the internet of health things," IEEE Access, vol. 6, pp. 13129-13141, 2018.

[13] J. Srinivas, A. K. Das, N. Kumar, and J. Rodrigues, "Cloud centric authentication for wearable healthcare monitoring system," IEEE Trans. Dependable Secure Comput., 2018.

[14] M. W. Moreira, J. J. Rodrigues, V. Korotaev, J. Al-Muhtadi, and N. Kumar, "A comprehensive review on smart decision support systems for health care," IEEE Systems Journal, vol. 13, no. 3, pp. 3536-3545, 2019.

[15] M. A. da Cruz, J. J. P. Rodrigues, J. Al-Muhtadi, V. V. Korotaev, and V. H. C. de Albuquerque, "A reference model for internet of things middleware," IEEE Internet of Things Journal, vol. 5, no. 2, pp. 871-883, 2018

[16] M. Awais, M. Raza, K. Ali, Z. Ali, M. Irfan, O. Chughtai, I. Khan, S. Kim, M. Ur Rehman, "An Internet of Things based bed-egress alerting paradigm using wearable sensors in elderly care environment", Sensors, vol. 19, no. 11, pp. 2498, 2019.

[17] S. Dash, A. Abraham, A. K. Luhach, J. Mizera-Pietraszko, and J. J. Rodrigues, "Hybrid chaotic firefly decision making model for Parkinson's disease diagnosis," Intl. J. Distributed Sensor Networks, vol. 16, no. 1, 2020.

[18] L. Naranjo, C. J. Pérez, J. Martín, Y. Campos-Roca, "A two-stage variable selection and classification approach for Parkinson's disease detection by using voice recording replications", Comput. Methods Programs Biomed., vol. 142, pp. 147-156, Apr. 2017.

[17] A. U. Haq et al., "Comparative analysis of the classification performance of machine learning classifiers and deep neural network classifier for prediction of parkinson disease", Proc. IEEE Int. Comput. Conf. Wavelet Active Media Technol. Inf. Process., pp. 101-106, Dec. 2018.

[20] M. Corzani, A. Ferrari, P. Ginis, A. Nieuwboer, and L. Chiari, "Motor Adaptation in Parkinson's disease during prolonged walking in response to corrective acoustic messages," Frontiers in Aging Neuroscience, vol. 11, p. 265, 2019.

[20] C. R. Pereira et al., "Handwritten dynamics assessment through convolutional neural networks: An application to parkinson's disease identification", Artif. Intell. Med., vol. 87, pp. 67-77, May 2018.

[22] B. Harel, M. Cannizzaro, P. J. Snyder, "Variability in fundamental frequency during speech in prodromal and incipient Parkinson's disease: A longitudinal case study", Brain Cogn., vol. 56, pp. 24-29, 2004.

[23] B. Sakar, M. Isenkul, C. Sakar, A. Sertbas, F. Gurgen, S. Delil, H. Apaydin, O. Kursun, "Collection and analysis of a Parkinson speech dataset with multiple types of sound recordings", IEEE Biomed. Health Informatics, vol. 17, no. 4, pp. 828-834, 2013.

[24] M. I. Razzak, M. Imran, G. Xu, "Big data analytics for preventive medicine", Proc. Neural Comput. Appl., pp. 1-35, Mar. 2019.

[25] A. Abós et al., "Discriminating cognitive status in Parkinson's disease through functional connectomics and machine learning", Sci. Rep., vol. 7, Mar. 2017

[26] N. Hassan, S. Gillani, E. Ahmed, I. Ibrar, M. Imran, "The role of edge computing in Internet of Things", IEEE Commun. Mag., vol. 56, no. 11, pp. 110-115, Nov. 2018.

[27] E. Ahmed et al., "Bringing computation closer toward the user network: Is edge computing the solution?", IEEE Commun. Mag., vol. 55, no. 11, pp. 138-144, Nov. 2017.

[28] M. Raza, H. Le-minh, N. Aslam, and S. Hussain, "A novel MAC proposal for critical and emergency communications in Industrial Wireless Sensor Networks," Comput. Electr. Eng, vol. 72, pp. 976-989, 2018.

[29] M. Raza et al., "Dynamic priority based reliable real-time communications for infrastructure-less networks", IEEE Access, vol. 6, pp. 67338-67359, 2018.

[30] A. Tsanas, M. A. Little, P. E. McSharry, L. O. Ramig, "Accurate telemonitoring of Parkinson's disease progression using non-invasive speech tests", IEEE Trans. Biomed. Eng., vol. 57, no. 4, pp. 884-893, Apr. 2010 .
[31] C. G. Goetz et al., "Testing objective measures of motor impairment in early Parkinson's disease: Feasibility study of an at-home testing device", Movement Disord., vol. 24, no. 4, pp. 551-556, 2009.

[32] K. M. Ghori, R. A. Abbasi, M. Awais, M. Imran, A. Ullah, and L. Szathmary, "Performance Analysis of Different Types of Machine Learning Classifiers for Non-Technical Loss Detection," IEEE Access, vol. 8, pp. 16033-16048, 2019.

[33] S. Rahman, M. Irfan, M. Raza, K. Moyeezullah Ghori, S. Yaqoob, and M. Awais, "Performance Analysis of Boosting Classifiers in Recognizing Activities of Daily Living," Intl. J. Environmental Research and Public Health, vol. 17, no. 3, p. 1082, 2020

[34] T. Chen, C. Guestrin, "XGBoost: A scalable tree boosting system", Proc. SIGKDD, pp. 785-794, 2016

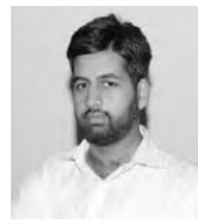

Dr Mohsin Raza is lecturer at Northumbria University, UK. Prior to this, he worked as a post-doctoral fellow (2018-19) at Middlesex University, UK, Junior lecturer (2010-12) and later as Lecturer (2012-15) in at Mohammad Ali Jinnah University, Pakistan, and Hardware Engineer (2009-10) at USS, Pakistan. His research interests include IoT, 5G, ITS, machine learning, Industry 4.0 and digital twins.

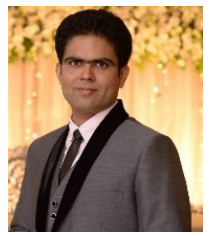

Dr. Muhammad Awais is a Research Fellow in Data Analytics and AI at University of Hull. His research interests are in data mining, signal processing, applied machine learning and deep learning to develop ICT (Information and communication Technologies) based systems for remote sensing, Interent of things, Industry 4.0 analytics, biomedical and health care domain.

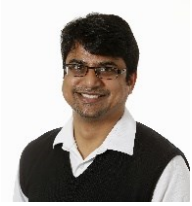

Dr Nishant Singh was awarded a $\mathrm{PhD}$ in Bio-Medical Technology, Birla Institute of Technology (BIT), India. Currently, he is a postdoc research fellow at the School of Psychology, University of Birmingham, UK. He worked as a Research Assistant at the Biomedical Instrumentation Laboratory, BIT, and later as a post-doc (2018-19) at the Computer Science Department, Middlesex University, UK.

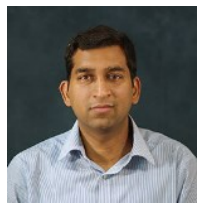

Dr Sajjad Hussain is Assistant Professor (Lecturer) in the School of Engineering, University of Glasgow. Previously, he served at the Capital University of Science and Technology, Pakistan as Associate Professor. He completed his masters from Supelec, Gif-sur-Yvette and PhD in 2009 from the University of Rennes 1, France.

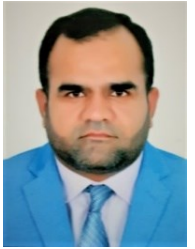

Muhammad Imran is an associate professor in the College of Applied Computer Science, King Saud University. His research interests include mobile and wireless networks, IoT, software-defined networking, cloud and edge computing, and information security. He has published several research papers in top journals. He serves as the Editor-in-Chief of EAI Transactions on Pervasive Health and Technology and Associate Editor for many top ranked international journals, such as IEEE Access, IEEE Communications Magazine, and Future Generation Computer Systems. 